\title{
Anfíbios do Parque Ambiental Chico Mendes, Rio Branco - Acre, Brasil
}

\author{
Nathocley Mendes Venâncio ${ }^{1 *}$ \\ Moisés Barbosa de Souza ${ }^{2}$ \\ Universidade Federal do Acre, Campus Universitário Áulio Gélio Alves de Souza \\ BR 364 km 04, CEP 69902-35, Rio Branco - AC, Brasil \\ ${ }^{1}$ Laboratório de Herpetologia \\ ${ }^{2}$ Centro de Ciências Biológicas e da Natureza \\ * Autor para correspondência \\ nathocley@gmail.com
}

Submetido em 16/07/2015

Aceito para publicação em 22/12/2015

\section{Resumo}

A anfibiofauna do Acre, apesar de pouco conhecida, é considerada uma das mais diversas. Este estudo teve por objetivo inventariar a fauna de anfíbios do Parque Ambiental Chico Mendes, fragmento florestal de 57 ha, localizado a $7 \mathrm{~km}$ do centro de Rio Branco. O estudo foi realizado entre agosto de 2008 e julho de 2010 , com a metodologia de busca visual e auditiva. Todas as trilhas e poças temporárias do parque foram inventariadas, onde registramos os indivíduos visualizados ou em atividade de vocalização. Foi encontrado um total de 51 táxons, distribuídos nas ordens: Anura, com 50 espécies, e Caudata, com uma espécie. Os anfíbios do parque mostraram sazonalidade quanto à reprodução, sendo os meses mais chuvosos aqueles com maior número de espécies em atividade reprodutiva.

Palavras-chave: Acre; Amazônia; Anfíbios; Fragmento florestal

\section{Abstract}

Amphibians in the Environmental Park Chico Mendes, Rio Branco - Acre, Brazil. Acre's amphibian fauna, although poorly known, is regarded as one of the most diversified. This study aimed to inventory the amphibian fauna in the Environmental Park Chico Mendes, a 57 ha forest fragment, located $7 \mathrm{~km}$ far from downtown Rio Branco. The study was conducted between August 2008 and July 2010, by using a visual and auditory search methodology. All tracks and temporary forest pools in the park were inventoried, where the individuals visualized or those using vocalization were registered. A total of 51 taxa were found, distributed into the orders: Anura, with 50 species, and Caudata, with 1 species. Amphibians in the park showed seasonality in reproduction, where the rainiest months were those with the highest number of species in reproductive activity.

Key words: Acre; Amazon rainforest; Amphibians; Forest fragment 


\section{Introdução}

Atualmente são conhecidas no mundo cerca de 7380 espécies de anfíbios (FROST, 2015), sendo a maior riqueza encontrada na região neotropical (DUELLMAN, 1988; BERTOLUCI, 1998). O Brasil é o país com a maior diversidade de anfíbios, com 1026 espécies (SEGALLA et al., 2014).

A anurofauna da Amazônia é considerada pouco conhecida (AZEVEDO-RAMOS; GALATTI, 2002). Mesmo considerada pouco conhecida, os estudos realizados no Acre mostram que essa é uma das regiões de maior diversidade de anfíbios do planeta, com 126 espécies registradas somente para a região do alto rio Juruá (SOUZA, 2009), no entanto, esse número já está ultrapassado, devido aos crescentes trabalhos realizados nessa região nos últimos anos, que resultaram em novos registro de várias espécies para o Acre (e.g., BERNARDE et al., 2010; 2011; VENÂNCIO et al., 2010; 2014a; 2014b; MELO-SAMPAIO; SOUZA, 2009; 2015) e na descrição de espécies novas (e.g., NUNESDE-ALMEIDA; TOLEDO, 2012; MELO-SAMPAIO et al., 2013).

Os inventários são ferramentas importantes para a conservação da Amazônia, pesquisas biológicas estimulam a descoberta de novas espécies e revelam áreas de endemismo e os níveis atuais de biodiversidade; consequentemente, os esforços de conservação podem ser apropriadamente dirigidos para o bioma (KRESS et al., 1998; SOARES-FILHO et al., 2006).

Tendo em vista a importância dos inventários, o presente estudo tem como objetivo inventariar a diversidade de anfíbios do Parque Ambiental Chico Mendes - PACM em Rio Branco - AC, Brasil, e estudar a relação destes com a precipitação ao longo do período estudado.

\section{Material e Métodos}

\section{Área de estudo}

O presente trabalho foi realizado em um fragmento florestal urbano de 57 ha, o Parque Ambiental Chico Mendes - PACM (Figura 1). Este é um jardim zoológico e está localizado a $7 \mathrm{~km}$ do centro da cidade de Rio Branco, (1002'11'S e $\left.67^{\circ} 47^{\prime} 43^{\prime \prime} \mathrm{W}\right)$ com cerca de $160 \mathrm{~m}$ de altitude, sua vegetação é composta por uma pequena parcela de mata primária, que sofreu corte seletivo de madeira. Nesse ambiente a floresta tem altura entre 30 e $40 \mathrm{~m}$, com a presença de algumas castanheiras (Bertholletia excelsa) e seringueiras (Hevea brasiliensis). Na maior parte da área, a floresta é composta de mata secundária (capoeira), tendo as árvores altura entre 5 e $15 \mathrm{~m}$, com algumas manchas de bambu (Guadua weberbaueri).

\section{Coleta de dados}

Os dados foram coletados entre agosto de 2008 e julho de 2010, entre as $18 \mathrm{~h}$ e $23 \mathrm{~h}$. A coleta foi feita por meio de uma visita semanal, totalizando quatro visitas mensais durante os meses secos, período de estiagem (abril a setembro), e duas visitas semanais, totalizando oito visitas mensais, no período chuvoso (outubro a março).

Foi utilizado o método de busca ativa visual e auditiva, que foram registrados todos os indivíduos visualizados e/ou em atividade de vocalização. Os ambientes estudados foram as trilhas dentro da floresta e as poças temporárias do interior da floresta, bordas e áreas abertas, que foram vistoriadas durante todas as visitas para a coleta de dados; dessa maneira, buscávamos sempre voltar aos mesmos ambientes amostrados, durante todo o período estudado. Indivíduos que não foi possível identificar em campo foram coletados, levados para o laboratório, identificados, sacrificados com pomada de lidocaína $50 \mathrm{mg}$, fixados em formalina a $10 \%$ e, após $48 \mathrm{~h}$, transferidos para álcool a 70\% e depositados na Coleção Herpetológica da Universidade Federal do Acre. O teste correlação de Pearson (ZAR, 1999) do programa Statistica 7.0 foi utilizado para calcular a correlação entre as espécies de anfíbios em atividade reprodutiva e a precipitação mensal. A nomenclatura utilizada no presente estudo seguiu Frost (2015).

O número de espécies em atividade reprodutiva foi determinado a partir das espécies que apresentavam machos vocalizando, fêmeas oocitadas e casais em 
FIGURA 1: Localização do Parque Ambiental Chico Mendes - PACM, na cidade de Rio Branco - AC, Brasil.

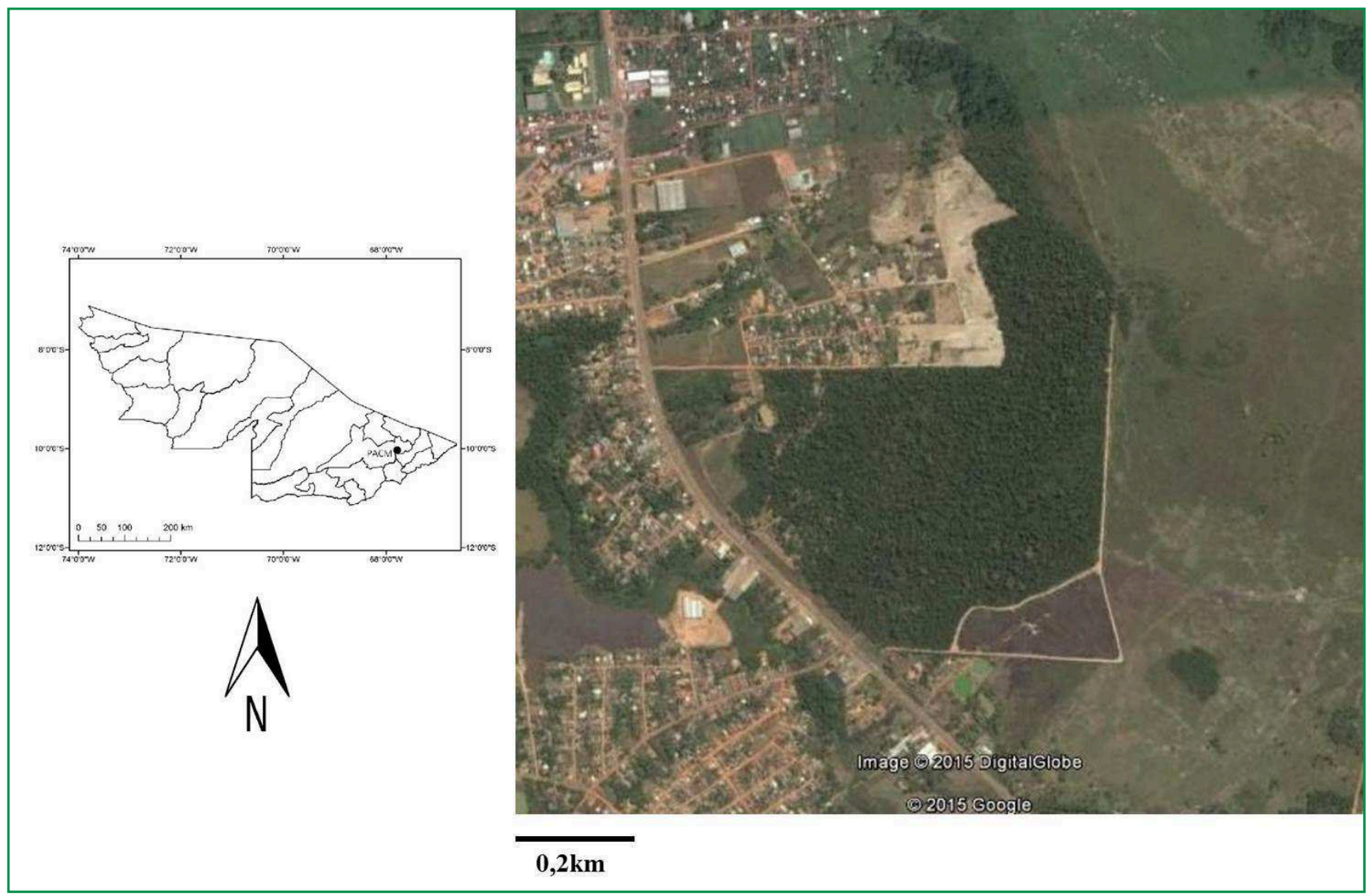

amplexo, contando-se o número de espécies em atividade reprodutiva para cada mês, durante todo o período de estudo. A precipitação mensal foi retirada de uma estação de coleta de dados pluviométricos, para a cidade de Rio Branco, localizada próxima à área de estudo (DUARTE, 2015).

A correlação existente entre o número de espécies em atividade reprodutiva e a precipitação mensal foi calculada com o auxílio do software Bio State 5.0, por meio de uma correlação linear de Pearson, já que os dados apresentaram curva de distribuição normal.

\section{Resultados}

Encontramos 51 espécies de anfíbios, distribuídos em duas ordens e 10 famílias. Ordem Anura, 50 espécies, distribuídas nas famílias: Aromobatidae (02), Bufonidae (03), Ceratophryidae (01), Craugastoridae (03), Dendrobatidae (01), Hylidae (25), Leptodactylidae (11), Microhylidae (03) e Pipidae (01). Ordem Caudata: Família Plethodontidae (01 espécie) (Tabela 1). Algumas das espécies encontradas no presente estudo estão representadas nas Figuras 2 a 6.

O maior número de espécies em atividade reprodutiva foi encontrado nos período entre novembro e março, coincidindo com o período chuvoso (Figura 7). Havendo uma correlação positiva entre o número de espécies reproduzindo e a precipitação mensal, durante os 24 meses de estudo na área amostrada ( $\mathrm{F}=16,39$; $\left.r^{2}=0,4009 ; \mathrm{p}=0,0005 ; \mathrm{n}=24\right)$. 
TABELA 1: Lista de espécies de anfíbios encontrados no Parque Ambiental Chico Mendes, Rio Branco - AC, entre agosto de 2008 a junho de 2010.

\section{TÁXONS}

ANURA (50)

\section{Aromobatidae (02)}

Allobates sp. 1

Allobates sp.2

Bufonidae (03)

Rhinella major (Müller \& Hellmich 1936)

Rhinella cf. margaritifera (Laurenti, 1768)

Rhinella marina (Linnaeus, 1758)

Ceratophryidae (01)

Ceratophrys cornura (Linnaeus, 1758)

Craugastoridae (03)

Pristimantis conspicillatus (Günther, 1858)

Pristimantis cf. fenestratus (Steindachner, 1864)

Pristimantis zimmermanae (Heyer\& Hardy 1991)

Dendrobatidae (01)

Ameerega hahneli (Boulenger, 1884)

Hylidae ( 25 )

Dendropsophus acreanus (Bokermann, 1964)

Dendropsophus bokermanni (Goin, 1960)

Dendropsophus brevifrons (Duellman \& Crump, 1974)

Dendropsophus leucophyllatus (Beireis, 1783)

Dendropsophus cf. minutus (Peters, 1872)

Dendropsophus parviceps (Boulenger, 1882)

Dendropsophus rhodopeplus (Günther, 1858)

Dendropsophus riveroi (Cochran \& Goin, 1970)

Dendropsophus sarayacuensis (Shreve, 1935)

Dendropsophus timbeba (Martins \& Cardoso, 1987)

Dendropsophus walfordi (Bokerman, 1962)

Dendropsophus xapuriensis (Martins \& Cardoso, 1987)

Hypsiboas calcaratus (Troschel, 1848)

Hypsiboas cinerascens (Spix, 1824)

Hypsiboas fasciatus (Günther, 1858)
Hypsiboas geographicus (Spix, 1824)

Hypsiboas lanciformis (Cope, 1871)

Osteocephalus taurinus (Steindachner, 1862)

Phyllomedusa camba De la Riva, 1999

Phyllomedusa palliata Peters, 1873

Scarthyla goinorum (Bokermann, 1962)

Scinax ruber (Laurenti, 1768)

Scinax garbei (Miranda-Ribeiro, 1926)

Sphaenorhynchus lacteus (Daudin, 1802)

Trachycephalus typhonius (Linnaeus 1758)

\section{Leptodactylidae (11)}

Adenomera andreae (Müller, 1923)

Adenomera hylaedactyla (Cope, 1868)

Engystomops freibergi (Donoso-Barros 1969)

Leptodactylus bolivianus Boulenger, 1898

Leptodactylus leptodactyloides (Andersson, 1945)

Leptodactylus didymus Heyer, García-Lopez \& Cardoso, 1996

Leptodactylus pentadactylus (Laurenti, 1768)

Leptodactylus petersii (Steindachner, 1864)

Leptodactylus rhodomystax Boulenger, 1884 “1883"

Leptodactylus wagneri (Peters, 1862)

Lithodytes lineatus (Schneider, 1799)

Microhylidae (03)

Chiasmocleis bassleri Dunn, 1949

Elachistocleis muiraquitan Nunes-de-Almeida and Toledo, 2012

Hamptophryne boliviana (Parker, 1927)

Pipidae (01)

Pipa pipa (Linnaeus, 1958)

CAUDATA (01)

Plethodontidae (01)

Bolitoglossa sp. 
FIGURA 2: A) Allobates sp. 1; B) Allobates sp. 2; C) Ameerega hahnelli; D) Rhinella cf. margaritifera; E) Rhinella marina; F) Rhinella major; G) Ceratophrys cornuta; H) Dendropsophus acreanus.
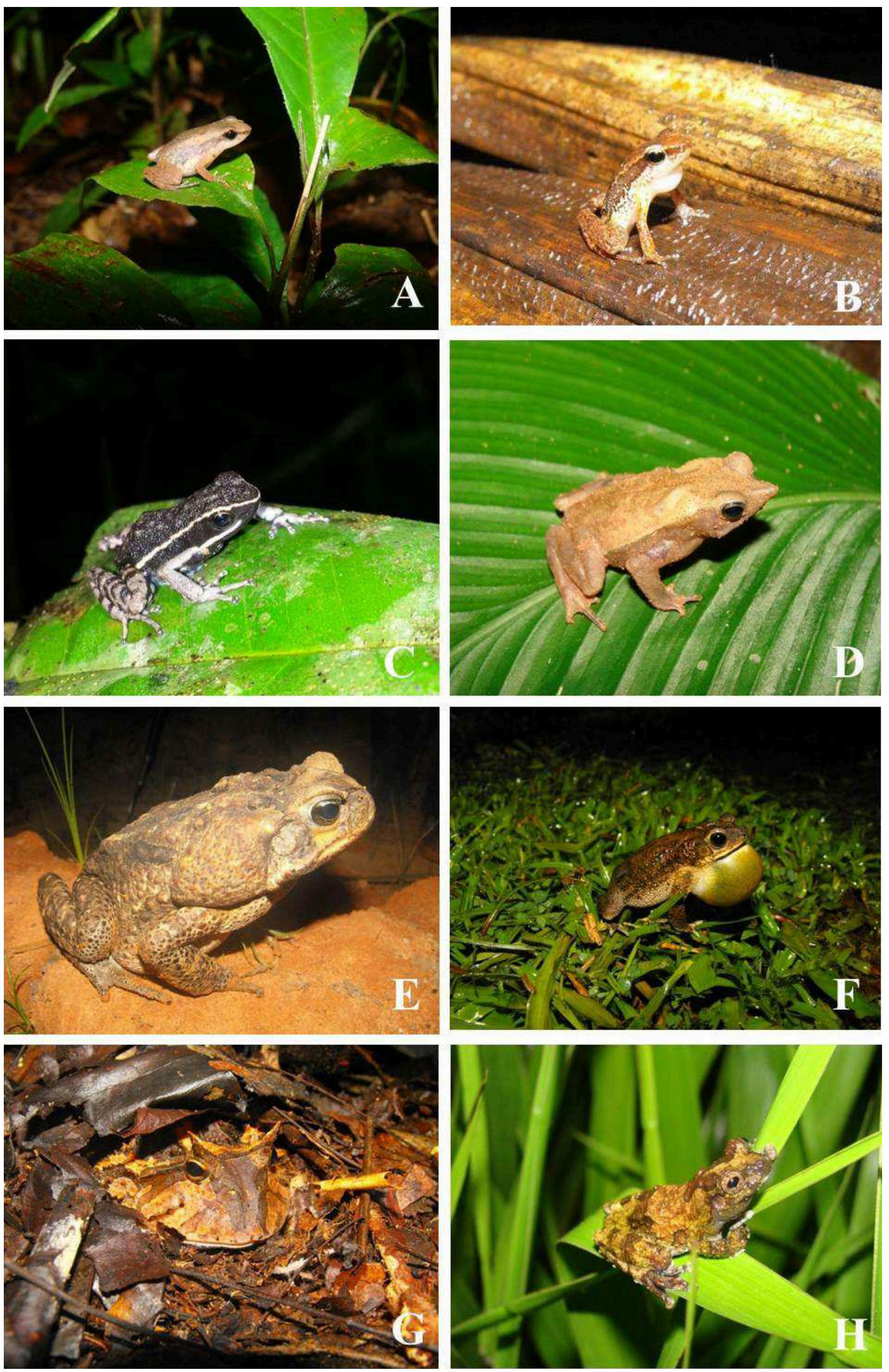
FIGURA 3: A) Dendropsophus brevifrons; B) Dendropsophus leucophyllatus; C) Dendropsophus parviceps; D) Dendropsophus rhodopeplus; E) Dendropsophus sarayacuensis; F) Dendropsophus timbeba; G) Dendropsophus xapuriensis; H) Hypsiboas calcaratus.
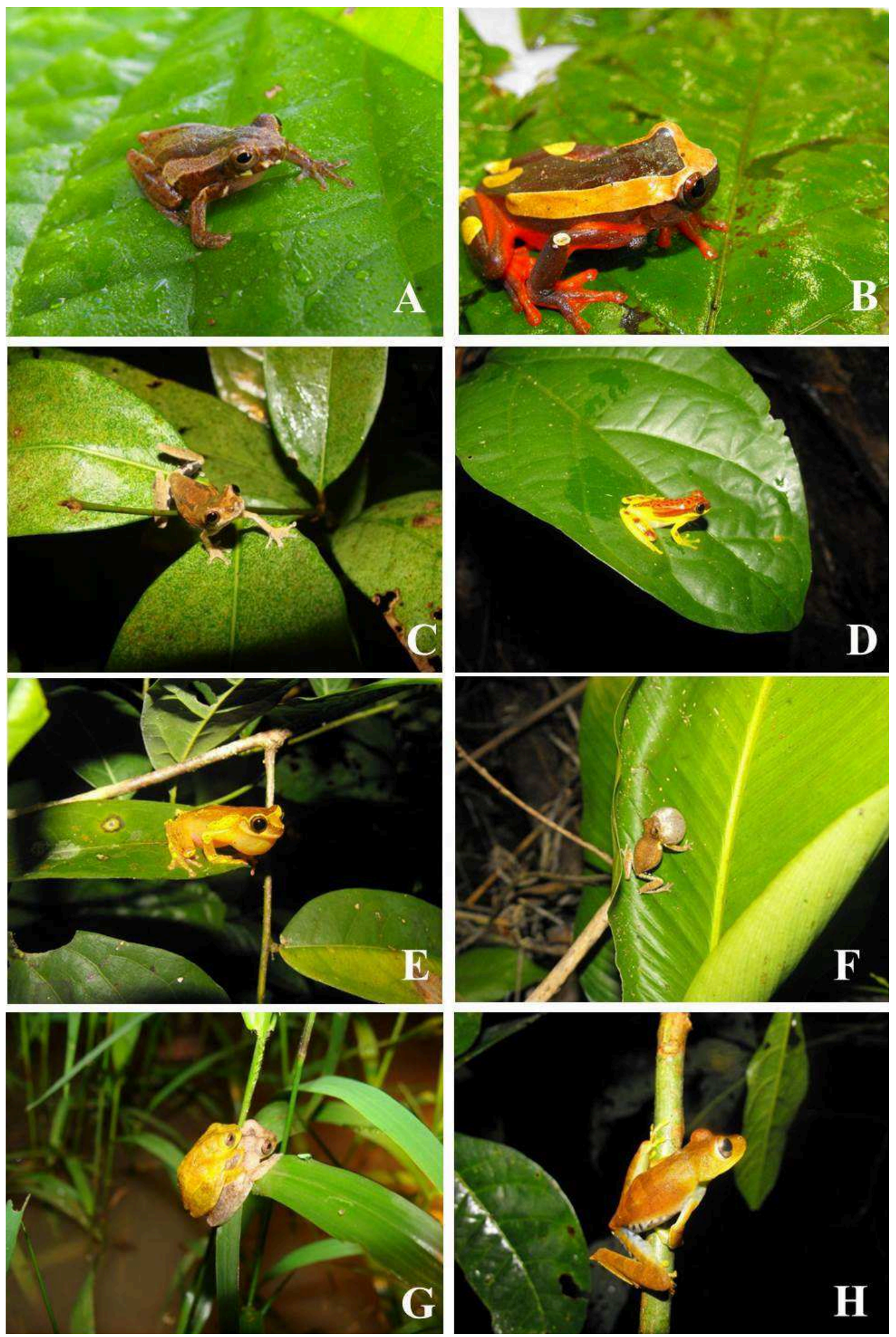
FIGURA 4: A) Hypsiboas cinerascens; B) Hypsiboas fasciatus; C) Hypsiboas geographicus; D) Hypsiboas lanciformis; E) Osteocephalus taurinus; F) Phyllomedusa camba; G) Phyllomedusa palliata; H) Engystomops freibergi.
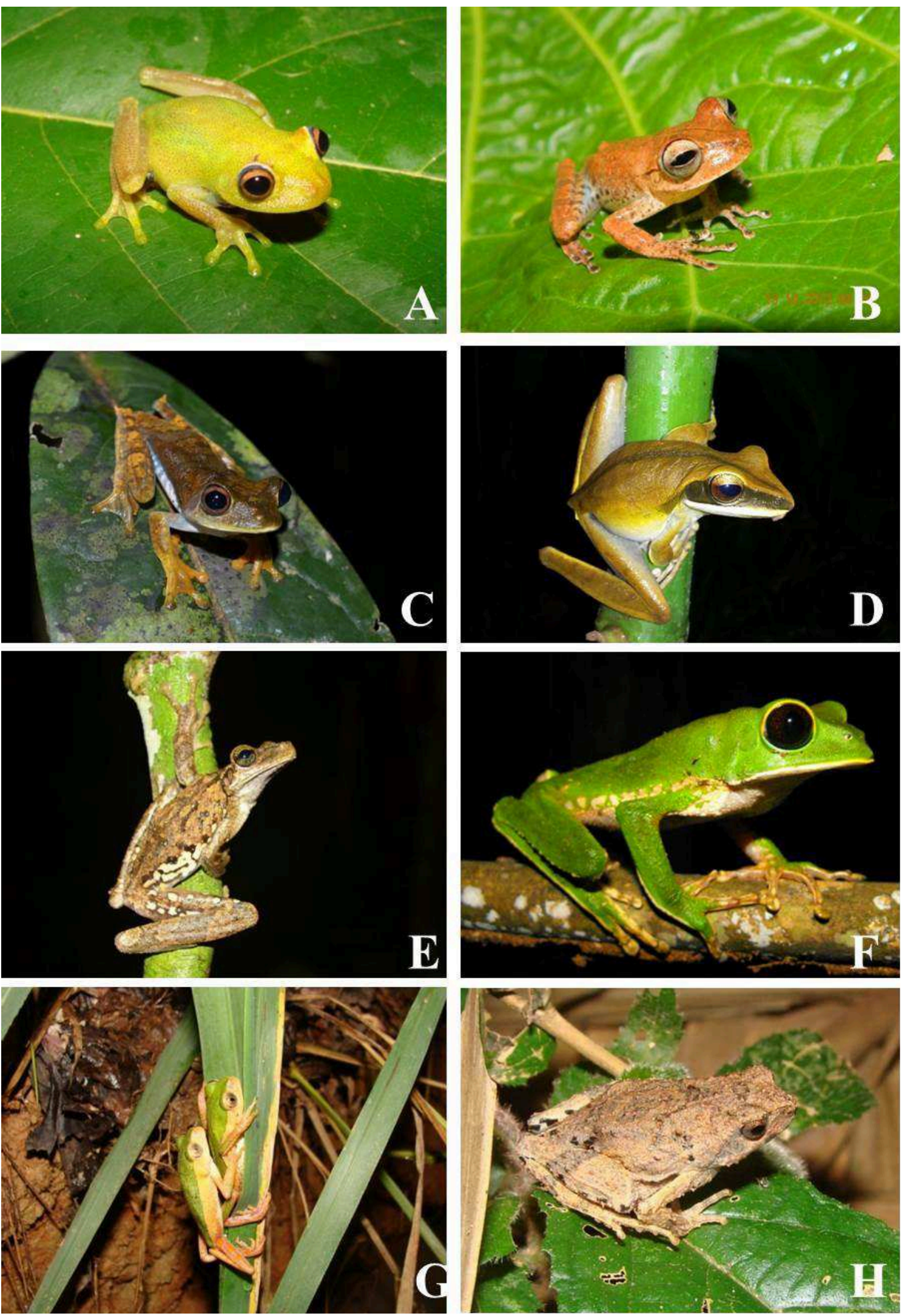
FIGURA 5: A) Leptodactylus bolivianus; B) Leptodactylus didymus; C) Lithodytes lineatus; D) Leptodactylus leptodactyoides; E) Leptodactylus pentadactylus; F) Leptodactylus petersii; G) Leptodactylus rhodomystax; H) Elachistocleis muiraquitan.
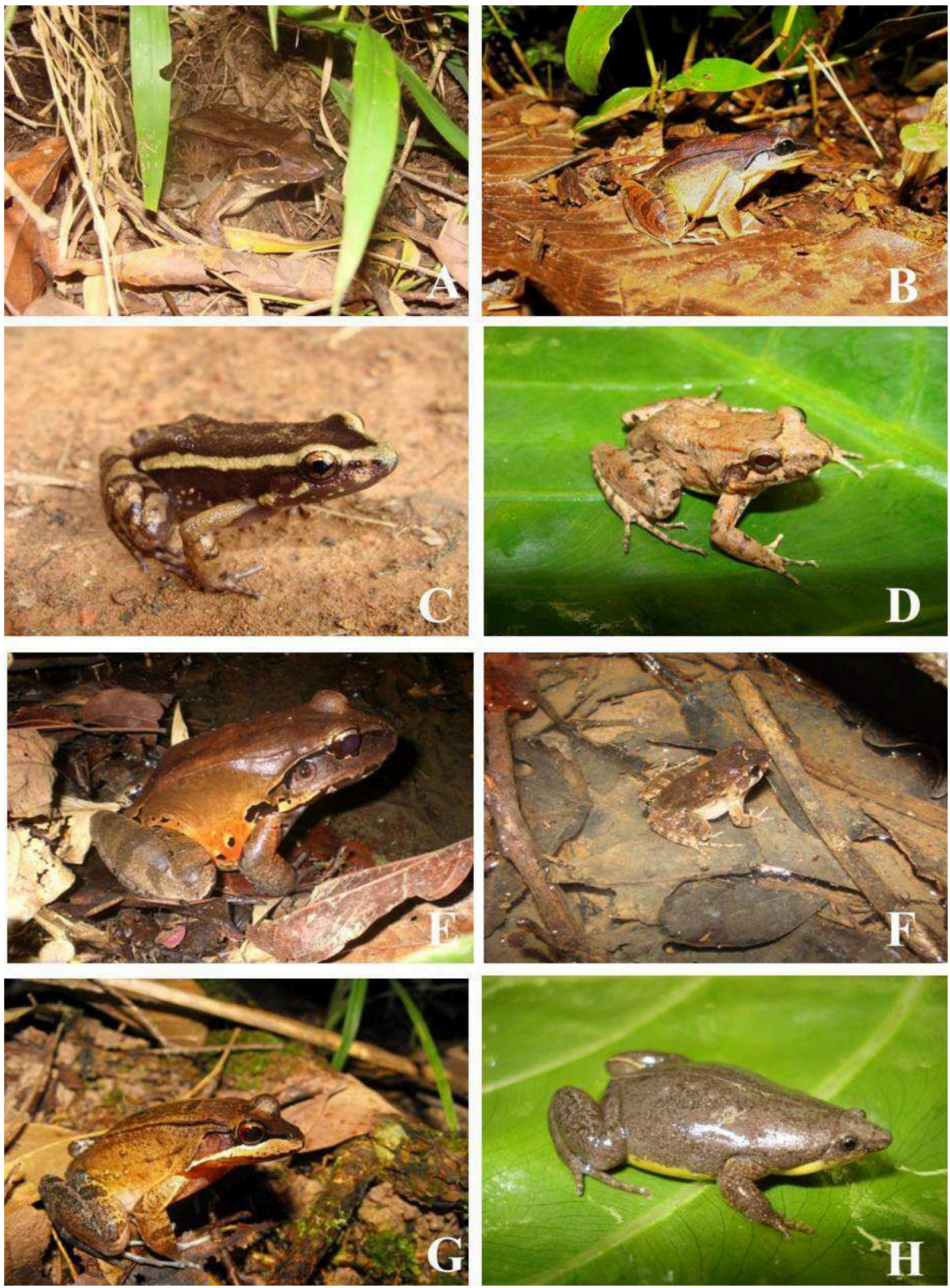
FIGURA 6: A) Hamptophryne boliviana; B) Pipa pipa; C) Bolitoglossa sp.
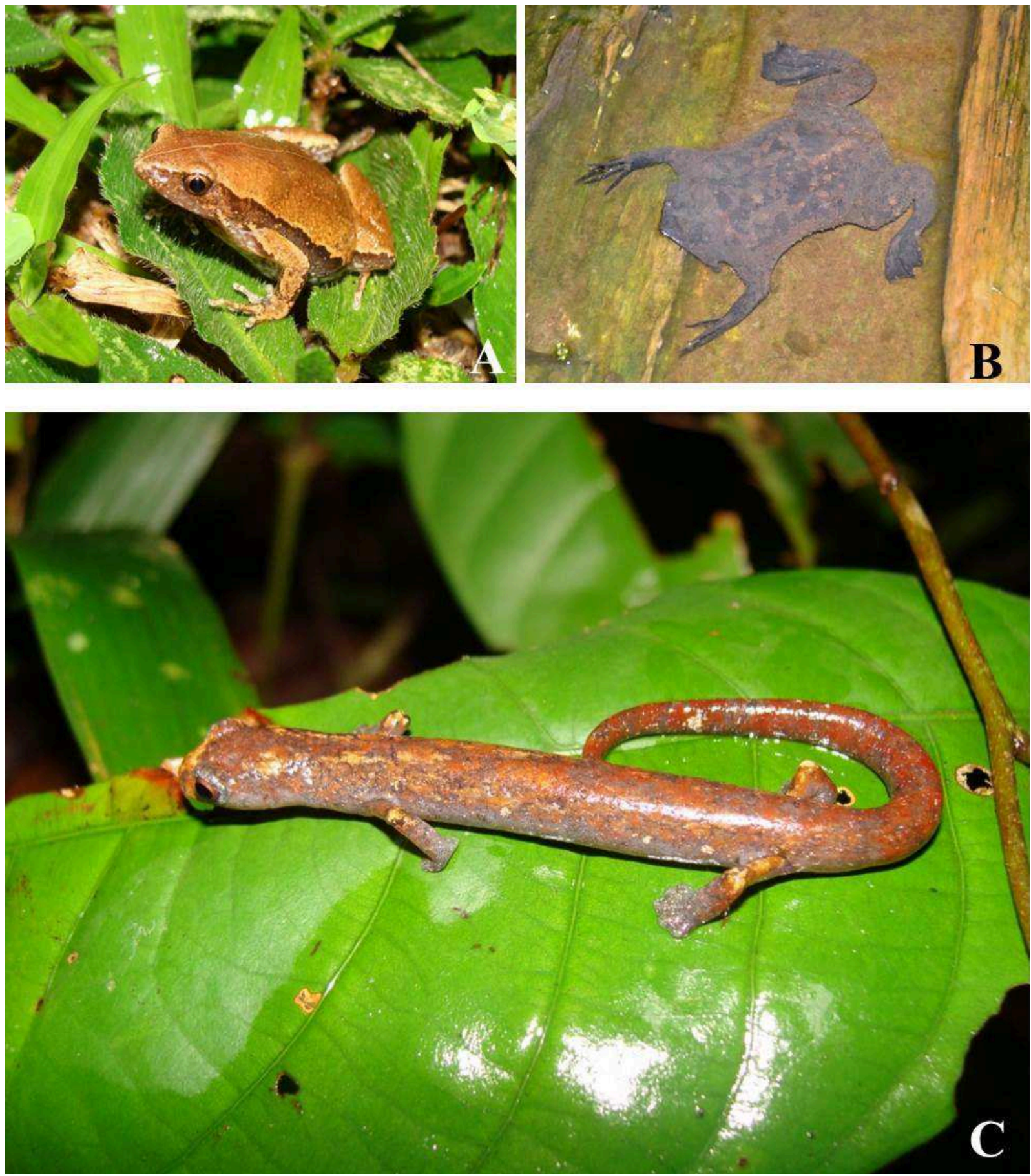
FIGURA 7: Associação entre o número de espécies reproduzindo (linha) e os meses (barras), durante o período estudado, no Parque Ambiental Chico Mendes, Rio Branco, Acre.

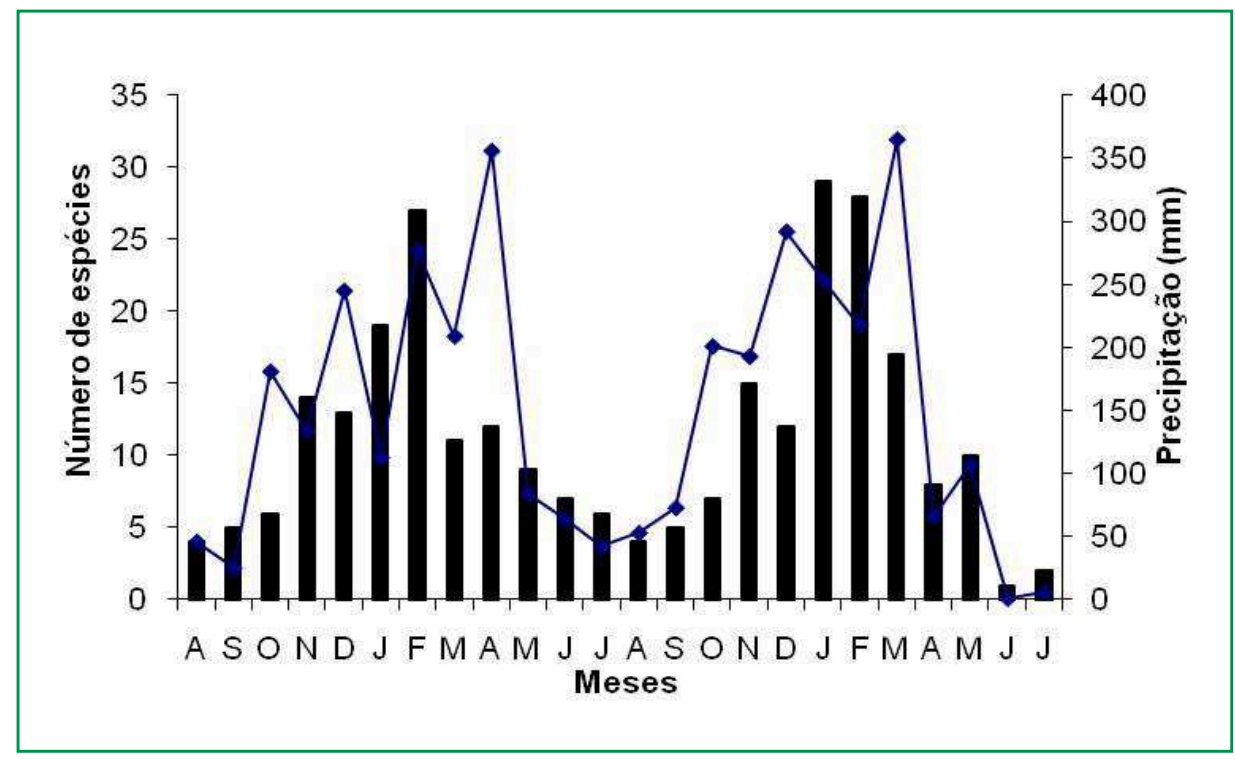

\section{Discussão}

Este estudo é de grande importância para o conhecimento dos anfíbios do PACM, pois, de acordo com Izecksohn e Carvalho-e-Silva (2001), as populações de anfíbios que habitam áreas urbanas sofrem ameaça de desaparecimento, principalmente devido à poluição e à destruição dos ambientes reprodutivos.

A presença do maior número de espécies encontradas nos meses de maior precipitação é algo comum nas regiões neotropicais, pois esse é um dos fatores que está fortemente correlacionado com a distribuição temporal dos anfíbios, estando a maioria deles restrita aos meses mais chuvosos do ano, que são encontrados em atividade reprodutiva (CARDOSO; MARTINS, 1987; HÖDL, 1990; SAENZ et al., 2006; BERNARDE, 2007).

A diversidade de anfíbios do parque é considerada alta quando comparada com estudos em outras regiões amazônicas e de maiores áreas, como Manaus/Reserva Ducke (50 espécies - LIMA et al., 2006), Espigão do Oeste (47 - BERNARDE, 2007), Alto Juruá (126 SOUZA, 2009), Purus River (43 - GORDO, 2003), Alto Purus (61 - RODRÍGUEZ, 2003), Cusco Amazônico (66 - DUELLMAN, 2005), Médio Madeira (41 - VOGT et al., 2007), Boca do Acre - AM (56 - FRANÇA;
VENÂNCIO, 2010), Reserva Extrativista Riozinho da Liberdade, AC (83 - BERNARDE et al., 2011), Reserva Extrativista do Rio Gregório (45 - PANTOJA; FRAGA, 2012) e a região de Carajás, no estado do Pará (71 PINHEIRO et al., 2012).

A alta riqueza de anfíbios em áreas com algum grau de antropização pode ser explicada pela colonização por espécies de áreas abertas (PEARMAN, 1997), mostrando que ambientes perturbados ainda são importantes enquanto unidades de conservação da fauna anfíbia.

\section{Agradecimentos}

Agradecemos ao CNPq, pela bolsa PIBIC ao autor sênior, à coordenadora do PACM Joseline Guimarães, por permitir nosso acesso ao PACM e ao amigo Herpetólogo Paulo Roberto Melo Sampaio, pela ajuda em algumas coletas de dados em campo.

\section{Referências}

AZEVEDO-RAMOS, C.; GALATTI, U. Patterns of amphibian diversity in Brazilian Amazônia: conservation implications. Biological Conservation, Boston, v. 103, p. 103-111, 2002.

BERNARDE, P. S. Ambientes e temporada de vocalização da anurofauna no município de Espigão do Oeste, Rondônia, Sudoeste 
da Amazônia - Brasil (Amphibia: Anura). Biota Neotropica, Campinas, v. 7, n. 2, p. 87-92, 2007.

BERNARDE, P. S.; MACHADO, R. A.; TURCI, L. C. B. Herpetofauna of Igarapé Esperança area in the Reserva Extrativista Riozinho da Liberdade, Acre-Brazil. Biota Neotropica, Campinas, v. 11, n. 3, p. 117-144, 2011.

BERNARDE, P. S.; MIRANDA, D. B.; ALBUQUERQUE, S.; TURCI, L. C. B. Amphibia, Anura, Hemiphractidae, Hemiphractus helioi Sheil and Mendelson, 2001: distribution extension in the state of Acre and second record for Brazil. Check List, Rio Claro, v. 6, n. 4, p. 491-492, 2010.

BERTOLUCI, J. Annual patterns of breeding activity in Atlantic rainforest anurans. Journal of Herpetology, Salt Lake City, v. 32, n. 4, p. 607-611, 1998.

CARDOSO, A. J.; MARTINS, J. E. Diversidade de anuros durante o turno de vocalizações, em comunidade neotropical. Papéis Avulsos de Zoologia, São Paulo, v. 36, n. 23, p. 279-285, 1987.

DUARTE, A. F. AcreBioVlima: an online reference. 2015. Disponível em: $<$ http://www.acrebioclima.net/>. Acesso em: 8 dez. 2015.

DUELLMAN, W. E. Patterns of species diversity in anuran amphibians in the American Tropics. Annals of the Missouri Botanical Garden, St. Louis, v. 75, p. 79-104, 1988.

DUELlMAN, W. E. Cusco Amazonico: the lives of amphibians and reptiles in an Amazonian Rainforest. Cornell: Comstock Books in Herpetology, 2005. 433 p.

FRANÇA, F. G. R.; VENÂNCIO, N. M. Reptiles and amphibians of a poorly known region in southwest Amazonia. Biotemas, Florianópolis, v. 23, n. 3, p. 71-84, 2010.

FROST, D. Amphibian species of the world: an online reference. 2015. Version 6.0. Disponível em: <http://research.amnh.org/ herpetology/amphibia/index.html>. Acesso em: 5 maio 2015.

GORDO, M. Os anfíbios anuros do Baixo Rio Purus/Solimões. In: DEUS, C. P.; SILVEIRA, R. da; PY-DANIEL, L. H. R. (Ed.). Piagaçu-Purus: bases científicas para a criação de uma reserva de desenvolvimento sustentável. Manaus: IDSM, 2003. p. 243-256.

HÖDL W. Reproductive diversity in Amazonian lowland frogs. Fortschritte der Zoologie, Stuttgart, v. 38, p. 41-60, 1990.

IZECKSOHN, E.; CARVALHO-E-SILVA, S. P. Anfíbios do município do Rio de Janeiro. Rio de Janeiro: Editora UFRJ, 2001.148 p.

KRESS, W. J; HEYER, W. R.; ACEVEDO, P.; CODDINGTON, J.; COLE, T. D.; ERWIN, L.; MEGGERS, B. J.; POGUE, M.; THORINGTON, R. W.; VARI, R. P.; WEITZMAN, M. J.; WEITZMAN S. H. Amazonian biodiversity: assessing conservation priorities with taxonomic data. Biodiversity and Conservation, New York, v. 7, n. 12, p. 1577-1587, 1998.

LIMA, A. P.; MAGNUSSON, W.; MENIN, M. Guia de sapos da Reserva Adolpho Ducke, Amazônia Central. Manaus: Áttema Design Editorial, 2006. 168 p.

MELO-SAMPAIO, P. R.; SOUZA, M. B. Ranitomeya biolat (bamboo poison frog): geographical distribution. Herpetological Review, St. Louis, v. 40 n. 4, p. 447, 2009.

MELO-SAMPAIO, P. R.; SOUZA, M. B. New and noteworthy distributional records of treefrogs (Anura) from southwestern Amazonia. Check List, Rio Claro, v. 11, n. 4, p. 1-7, 2015.

MELO-SAMPAIO, P. R.; SOUZA, M. B.; PELOSO, P. L. V. A new, riparian, species of Allobates Zimmermann and Zimmermann, 1988
(Anura: Aromobatidae) from southwestern Amazonia. Zootaxa, Auckland, v. 3716, n. 3, p. 336-348, 2013.

NUNES-DE-ALMEIDA, C. H. L.; TOLEDO, L. F. A new species of Elachistocleis Parker (Anura: Microhylidae) from the State of Acre, Northern Brazil. Zootaxa, Auckland, v. 3424, p. 43-50, 2012.

PANTOJA, D. L.; FRAGA, R. Herpetofauna of the Reserva Extrativista do Rio Gregório, Juruá Basin, southwest Amazonia, Brazil. Check List, Rio Claro, v. 8, n. 3, p. 360-364, 2012.

PEARMAN, P. B. Correlates of amphibian diversity in an altered landscape Amazonian Ecuador. Conservation Biology, San Francisco, v. 11, n. 5, p. 1211-1225, 1997.

PINHEIRO, L. C.; BITAR, Y. O. C.; GALATTI, U.; NECKELOLIVEIRA, S.; SANTOS-COSTA, M. C. Amphibians from southeastern state of Pará: Carajás Region, northern Brazil. Check List, Rio Claro, v. 8, n. 4, p. 693-702, 2012.

RODRÍGUEZ, L. O. Anfibios y reptiles de la region de Alto Purús. In: PITMAN, R. L.; PITMAN, N.; ÁlVAREZ, P. (Ed.). Alto Purús: biodiversidad, conservación y manejo. Lima: Center for Tropical Conservation, 2003. p. 89-96.

SAENZ, D.; FITZGERALD, L. A.; BAUM, K. A.; CONNER, R. $\mathrm{N}$. Abiotic correlates of anuran calling phenology: the importance of rain, temperature, and season. Herpetological Monographs, Lawrence, v. 20, p. 64-82, 2006.

SEGALlA, M. V.; CARAMASCHI, U.; CRUZ, C. A. G.; GARCIA, P. C. A.; GRANT, T.; HADDAD, C. F. B.; LANGONE, J. Brazilian amphibians - List of species. 2014. Disponível em: $<$ http://www.sbherpetologia.org.br/images/LISTAS/2014.02-07Mudanças Taxonomicas.pdf $>$. Acesso em: 8 jul. 2015.

SOARES-FILHO, B. S.; NEPSTAD, D. C.; CURRAN, L. M.; CERQUEIRA, G. C.; GARCIA, R. A.; RAMOS, C. A.; VOLL, E.; Mc DONALD, A.; LEFEBVRE, P.; SCHLESINGER, P. Modelling conservation in the Amazon basin. Nature, London, v. 440, n. 7083, p. 520-523, 2006.

SOUZA, M. B. Anfíbios: Reserva Extrativista do Alto Juruá e Parque Nacional da Serra do Divisor, Acre. Campinas: IFCH, 2009. $77 \mathrm{p}$.

VENÂNCIO, N. M.; LIMA, A. P.; SOUZA, M. B.; MAGNUSSON, W. E. Between-year consistency of anuran assemblages in temporary ponds in a deforested area in Western Amazonia. Herpetological Journal, Montrose, v. 24, p. 155-160, 2014a.

VENÂNCIO, N. M.; MOURA, A. L. B.; BRITO, T. F.; MELO, T. M.; SOUZA, M. B. First record of Cruziohyla craspedopus (Anura: Hylidae) for the state of Acre, with an updated distribution map. Herpetology Notes, Pisa, v. 7, p. 479-480, 2014b.

VENÂNCIO, N. M.; SOUZA, M. B.; FRANÇA, F. G. R. Amphibia, Anura, Leptodactylidae, Leptodactylus didymus Heyer, GarcíaLopez and Cardoso, 1996: distribution extension and geographic distribution map. Check List, Rio Claro, v. 6, n. 4, p. 646-647, 2010. VOGT, R. C.; FERRARA, C. R.; BERNHARD, R.; CARVALHO, V. T.; BALENSIEFER, D. C.; BONORA, L.; NOVELLE, S. M. H. Herpetofauna. In: PY-DANIEL, R. L.; DEUS, C. P.; HENRIQUES, A. L.; PIMPÃO, D. M.; RIBEIRO, O. M. (Org.). Biodiversidade do Médio Madeira: bases científicas para propostas de conservação. Manaus: INPA, 2007. p. 127-143.

ZAR, J. H. Biostatistical analysis. New Jersey: Prentice Hall, 1999. 663 p. 